\title{
Mitochondrial transcription factor A (TFAM) is upregulated in glioma
}

\author{
HYUNJI LEE $^{1 *}$, JISOO PARK $^{1 *}$, QUANGDON TRAN ${ }^{1}$, DOHOON KIM $^{1}$, \\ YOUNGEUN HONG ${ }^{1}$, HYEONJEONG CHO ${ }^{1}$, SO HEE KWON ${ }^{2}$, \\ DEREK BRAZIL $^{3}$, SEON-HWAN KIM $^{4}$ and JONGSUN PARK ${ }^{1}$ \\ ${ }^{1}$ Department of Pharmacology and Medical Science, Metabolic Diseases and Cell Signaling Laboratory, \\ Research Institute for Medical Sciences, College of Medicine, Chungnam National University, Daejeon 35015; \\ ${ }^{2}$ College of Pharmacy, Yonsei Institute of Pharmaceutical Sciences, Yonsei University, Incheon 21983, \\ Republic of Korea; ${ }^{3}$ Centre for Experimental Medicine, Queen's University Belfast, Belfast, BT9 7BL, Northern Ireland; \\ ${ }^{4}$ Department of Neurosurgery, Institute for Cancer Research, College of Medicine, \\ Chungnam National University, Daejeon 35015, Republic of Korea
}

Received August 20, 2016; Accepted April 4, 2017

DOI: $10.3892 / \mathrm{mmr} .2017 .6467$

\begin{abstract}
Mitochondrial transcription factor A (TFAM), which was initially discovered as a transcription factor for mitochondrial DNA, has known to be critical for the regulation of mitochondrial DNA. However the possible involvement of TFAM in cancer is largely unknown. In this study, we have provided some evidence that TFAM may have a potential role in brain tumor. Western blot analysis with anti-TFAM antibody indicated that TFAM is overexpressed in glioblastoma cell lines including U87MG and U251MG. Transcriptome profiling of U87MG and U251MG cells by using deep-sequencing revealed that TFAM transcripts were upregulated in these cells compared to its of cerebral cortex. Confocal microscopic analysis of U251MG cells with anti-TFAM antibody showed that TFAM is located to the dot-like structure close to nucleus, probably mitochondria and endosome. Immunohistochemical analysis of glioma tissue specimens indicated that TFAM is
\end{abstract}

Correspondence to: Dr Seon-Hwan Kim, Department of Neurosurgery, Institute for Cancer Research, College of Medicine, Chungnam National University, 282 Munhwa-Ro, Jung-Gu, Daejeon 35015, Republic of Korea

E-mail: neons@cnu.ac.kr

Dr Jongsun Park, Department of Pharmacology and Medical Science, Metabolic Diseases and Cell Signaling Laboratory, Research Institute for Medical Sciences, College of Medicine, Chungnam National University, 266 Munhwa-Ro, Jung-Gu, Daejeon 35015, Republic of Korea

E-mail: insulin@cnu.ac.kr

*Contributed equally

Key words: TFAM, mitochondria, glioma, biomarker highly upregulated. Bioinformatical analysis with Rembrandt knowledgebase also supported that TFAM mRNA is upregulated in glioma patients. Taken together, the results presented in this study obviously provided the evidence that TFAM was upregulated in glioma cell line and glioma tissue specimens. Therefore TFAM may be a novel diagnostic marker and therapeutic target for glioma and other cancer.

\section{Introduction}

Malignant gliomas are the most common primary brain tumors, and glioblastomas are among the most aggressive form of human cancers (1). Astrocytic tumors are the most common glial neoplasms, with an annual incidence of $3-4 / 100,000$ populations, and roughly $80 \%$ are glioblastomas. Genetic defects are shared in GBM (2). GBM is a malignant brain tumor typically resulting in death of the patient within one year following diagnosis (3). Angiogenesis is a hallmark of cancer. Tumor cells can initiate angiogenesis, by firstly progressing an angiogenic phenotype through angiogenic switch (4). Their characteristics are a solid linkage of extremely circuitous and large-diameter vessels (5). The tumor vasculature is functionally unusual and this increased vessel permeability can lead to vasogenic edema and hemorrhages (6). As the vasculature in GBMs is common with a seriously disordered vessel type, this may also limit the ability of radio- and chemotherapy by blocking blood flow (7). Accumulated preclinical and clinical reports have provided innovative molecular targets, which may improve the therapeutic advantage from anti-angiogenic strategies (8). At this time point, the promising drug for targeting vascular endothelial growth factor (VEGF) is bevacizumab (BEV), a recombinant antibody to block human VEGF-A (9). Nevertheless, the reported positive effect of BEV treatment may be due to imaging restrictions causing from reduced neoangiogenesis and reduced vascular permeability at least in part, thus resulting to an obvious but debatable reduction 
in the contrast-enhancing tumor volume (10). The phase III trials with avastin and radiation therapy has been performed to evaluate the effectiveness of temozolomide (TMZ)-based radio-chemotherapy with the BEV treatment for GBM patients (11). The case study of avastin treatment (AVAglio) for GBM patients indicated a significant improvement of progression-free survival (PFS) by 4.4 months with BEV co-treatment whereas radiation therapy alone group (RTOG) with BEV co-treatment failed to show significant benefits in terms of PFS and overall survival. However, this PFS benefit did not improve the overall survival (12). Hence it is critical to find the new target for GBM patient in clinics with the exception of the TMZ and VEGF targeting drug. Mitochondrial transcription factor A (TFAM) is a main regulator of mitochondrial transcription in mammals. Its expression level correlate to mtDNA content and gene expression (13). Also TFAM has a function in mitochondrial DNA replication, since transcription generates an RNA primer required for initiation of mtDNA replication (14). Abnormal function of mitochondria has been found to be connected to many metabolic disorders, including cancer (15). Therefore the putative role of TFAM in glioblastoma was investigated since the novel therapeutic strategy is urgently needed in brain cancer. In this study, we have provided the clear evidence to support the upregulation of TFAM in glioblastoma cell lines and the tissue from glioma patients. This information will provide critical knowledge for better understanding the regulatory mechanism of cancer as well as a promising therapeutic strategy for brain cancer.

\section{Materials and methods}

Antibodies and reagents. Anti-TFAM antibody and Anti-actin antibodies were purchased from Sigma-Aldrich (St. Louis, MO, USA). Horseradish peroxidase-conjugated anti-mouse IgG or anti-rabbit IgG secondary antibodies were purchased from Komabiotech (Seoul, Korea).

Cell culture. The glioblastoma cells (U87-MG, U251-MG, U343-MG and U373-MG) were maintained in medium (RPMI) supplemented with 10\% FBS, 25 mM HEPES (Thermo Fisher Scientific, Waltham, MA, USA), $1 \%$ antibiotics-antimycotics (Life Technologies, Carlsbad, CA, USA).

Immunoblot analysis. The western blot analysis was performed as previously described (16-18). Briefly, cells were placed on ice and extracted with lysis buffer containing $50 \mathrm{mM}$ Tris-HCl, pH 7.5, 1\% v/v Nonidet P-40, 120 mM NaCl, 25 mM sodium fluoride, $40 \mathrm{mM} \beta$-glycerol phosphate, $0.1 \mathrm{mM}$ sodium orthovanadate, $1 \mathrm{mM}$ phenylmethylsulfonyl fluoride, $1 \mathrm{mM}$ benzamidine, and $2 \mu \mathrm{M}$ microcystin-LR. Lysates were centrifuged for $15 \mathrm{~min}$ at $12,000 \mathrm{x} \mathrm{g}$. The cell extracts were resolved by $10-15 \%$ SDS-PAGE, and transferred to Immobilon-P membranes (Millipore). The filters were blocked for $1 \mathrm{~h}$ in 1X Tris-buffered saline buffer (TBS; $140 \mathrm{mM} \mathrm{NaCl}, 2.7 \mathrm{mM}$ $\mathrm{KCl}, 250 \mathrm{mM}$ Tris- $\mathrm{HCl}, \mathrm{pH} 7.4)$, containing 5\% skimmed milk and $0.2 \%$ Tween-20, followed by an overnight incubation with the anti-TFAM and anti-actin antibodies diluted 1,000 -fold at $4^{\circ} \mathrm{C}$. The secondary antibody was horseradish peroxidase-conjugated anti-mouse $\operatorname{IgG}$ or anti-rabbit IgG
(Komabiotech), diluted 5,000-fold in the blocking buffer. The detection of protein expression was visualized by enhanced chemiluminescence, according to the manufacturer's instructions (Thermo Fisher Scientific).

Real-time quantitative reverse transcription-polymerase chain reaction ( $q R T-P C R)$. Total RNA was extracted from frozen tissue samples or from cells using the PureHelix RNA Extraction Solution (Nanohelix, Korea). The cDNA was synthesized from total RNA with the SuperScript III First-Strand Synthesis system for qRT-PCR (Invitrogen, Grand Island, NY, USA). The qRT-PCR measurement of individual cDNAs was performed using SYBR-Green dye to measure duplex DNA formation with the StepOne Plus real-time PCR system (Invitrogen) and normalized to the expression of glyceraldehyde 3-phosphate dehydrogenase (GAPDH) RNA. The following primers were used in the qRT-PCR ( $\mathrm{F}$, forward; R, reverse); human TFAM, F-5'-GGCA AGTTGTCCAAAGAA ACC/R-5'-GCATCTGGGTTCTGAG CTTTA; human GAPDH, F-5'-TCG ACA GTC A GC CGC A TC T TC TTT/R-5'-TACGACCAAATCCGTTGACTCCGA.

RNA sequencing and RNA-Seq data analysis. Total RNA of U87-MG, U251-MG and normal brain was extracted using TRIzol reagent (Invitrogen) following the manufacturer's procedures. The total RNA quantity and purity were analysis of Bioanalyzer 2100 and RNA 6000 Nano LabChip kit (Agilent, Santa Clara, CA, USA). Roughly $10 \mu \mathrm{g}$ of total RNA was subjected to isolate Poly(A) mRNA with poly-T oligo attached magnetic beads (Invitrogen). Following purification, the mRNA is fragmented into small pieces using divalent cations under raised temperature. Then the cleaved RNA fragments were reverse-transcribed to create the final cDNA library in accordance with the protocol for the mRNA-Seq sample preparation kit (Illumina). The average insert size for the paired-end libraries was $300 \mathrm{bp}( \pm 50 \mathrm{bp})$. Next we performed the paired-end sequencing on an Illumina HiSeq 2000 system at Macrogen (Seoul, Korea) following the vendor's recommended protocol. For each sample, sequenced reads were aligned to the UCSC human reference genome (19) using the Tophat package (20), which initially removes a portion of the reads based on quality information accompanying each read and then maps the reads to the reference genome. FPKM (fragments per kilobase of exon per million fragments mapped) were calculated to compare the expression level of TFAM mRNA variants in each sample.

Confocal imaging analysis and indirect immunofluorescence. U251-MG cells were grown on glass coverslips until they were $50-70 \%$ confluent. After $24 \mathrm{~h}$, the cells were fixed in $4 \%$ paraformaldehyde at room temperature for $10 \mathrm{~min}$ and permeabilized in $0.2 \%$ Triton $\mathrm{X}-100$ for $5 \mathrm{~min}$ at room temperature. Then cells were incubated in blocking buffer containing 5\% bovine serum albumin (Sigma) in $1 \mathrm{X}$ TBS for $1 \mathrm{~h}$ at $37^{\circ} \mathrm{C}$. The rabbit polyclonal anti-TFAM was diluted 200 -fold for primary antibody and incubated for overnight. The secondary antibody, FITC-conjugated anti-rabbit antibody (BD Biosciences) was used. After appropriate rinsing, cover slips were mounted with Vectashield (Vector Laboratories) and visualized using a Zeiss confocal microscope. 

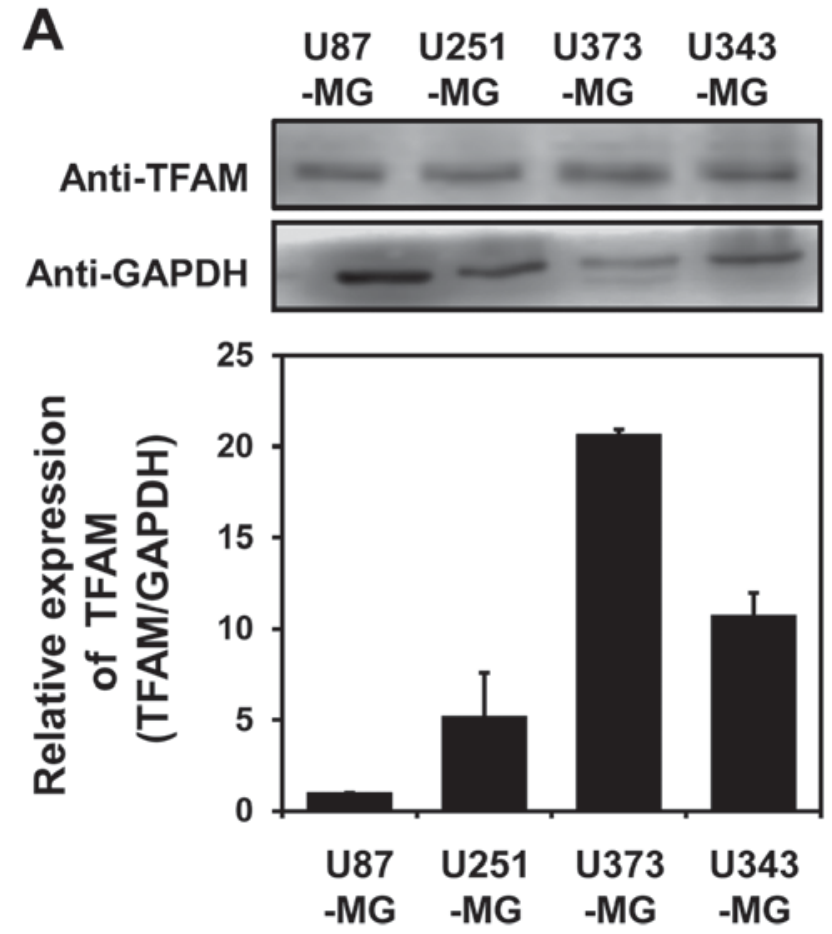

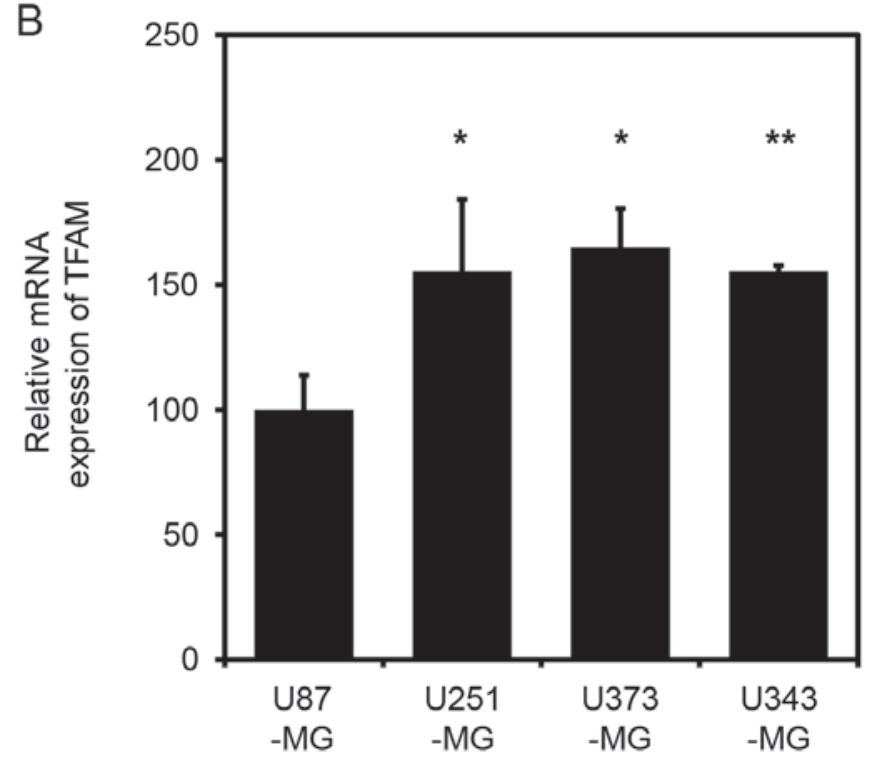

Figure 1. TFAM expression in glioblastoma (GBM) cell lines. (A) For western blot analysis with anti-TFAM and anti-actin antibodies, cell lysates was isolated from 4 established GBM cell lines (U87-MG, U251-MG, U373-MG and U343-MG). The results are representative of 3-independent experiments (top panel). Relative density was obtained by densitometry of the corresponding immunoblot data. Relative and statistical differences of TFAM expression were determined by normalizing values for actin in each lane and set the values for U87-MG as 1 (bottom panel). The results are presented as means \pm SD of three independent experiments. (B) Extracted total RNA from each GBM cell line was analyzed using human TFAM specific primer sets by real-time quantitative reverse transcription-polymerase chain reaction (qRT-PCR), as described in the Materials and methods section. The results are presented as means \pm SD of three independent experiments. ${ }^{*} \mathrm{P}<0.05,{ }^{* *} \mathrm{P}<0.01$.

Immunohistochemistry. The analysis of immunohistochemistry was performed as the described previously (21). A human cancer tissue array slide with paraffin sections was purchased from Bio Max (US Biomax Inc). Histostain-Plus kits (Zymed Laboratories Inc.) were used in accordance with the manufacturer's instructions for the immunohistochemistry of tissue array. Briefly, paraffin sections were deparaffinized with xylene and rehydrated in a graded series of ethanol. The slide was submerged in peroxidase quenching solution for $10 \mathrm{~min}$. After it was washed twice with PBS for $5 \mathrm{~min}$, it was added with 2 drops of reagent A for blocking and incubated for $30 \mathrm{~min}$. Following two washes with PBS, the primary antibody, anti-TFAM antibody, was applied at $4^{\circ} \mathrm{C}$ for overnight. Then biotinylated secondary antibody, reagent $\mathrm{B}$, was added after rinsing with PBS. It was incubated at room temperature for $1 \mathrm{~h}$. It was rinsed with PBS and dropped with enzyme conjugated reagent $C$. After it was washed with PBS, DAB chromogen, and a mixture of reagent $\mathrm{D} 1, \mathrm{D} 2$, and $\mathrm{D} 3$, it was dropped, and signals were observed with a florescence microscope. Then the reaction was stopped with distilled water, and pictures were taken with a microscope.

Bioinformatics data set. Glioma data sets and corresponding clinical data were downloaded from the publicly available databases (446 cases from the Repository of Molecular Brain Neoplasia Data (REMBRANDT; http://www.betastasis.com/ glioma/rembrandt/). Normal, $n=21$; GBM, $n=214$; oligodendroglioma, $\mathrm{n}=66$; astrocytomas, $\mathrm{n}=145$.
Statistical analysis. Data are expressed as the mean \pm SD from at least three separate experiments performed triplicate. The differences between groups were analyzed using a Student's $t$-test and $\mathrm{P}<0.05$ was considered significant, and $\mathrm{P}<0.01$ was highly significant compared with corresponding control values. Statistical analyses were carried out using SPSS software ver. 13.0 (SPSS Inc., Chicago, IL, USA).

\section{Results}

Enhancement of TFAM expression in glioblastoma cell lines. In order to investigate the putative roles of TFAM in brain cancer, western blot analysis with anti-TFAM antibody were performed. As shown in Fig. 1A, the protein expression of TFAM is markedly increased especially in U343-MG cells and U373-MG cells compared to other cells. Analysis of quantitative real-time PCR (qRT-PCR) with glioblastoma cell lines also showed that mRNA level of TFAM is elevated in U251-MG cells, U343-MG cells and U373-MG cells (Fig. 1B).

Transcriptional induction of TFAM mRNA in U87-MG cells and U251-MG cells. Based on the observation of Fig. 1A and B, the mRNA levels of TFAM were also monitored by using RNA sequencing of glioblastoma cell lines. Total RNA were isolated from two cell lines (U87-MG and U251-MG) which showed the low expression of TFAM by western blot analysis and normal brain for the comparison of TFAM expression. Roughly $10 \mu \mathrm{g}$ of total RNA was further isolated to mRNA. 


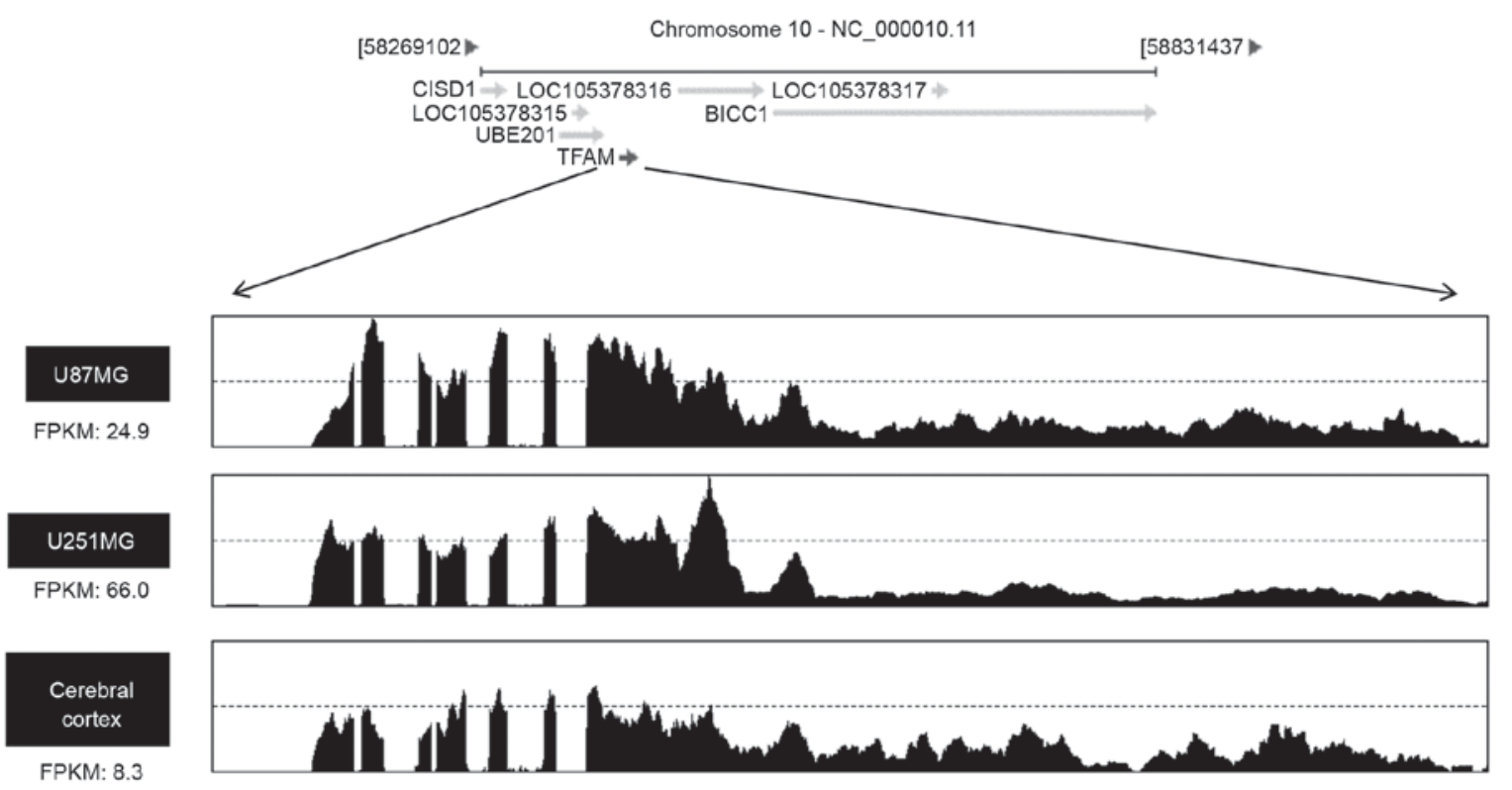

Figure 2. Relative changes of TFAM transcripts from GBM cells in standard RNA-seq data. Total RNA were isolated from two GBM cell lines (U87-MG and U251-MG) and normal brain tissue. These samples were further analyzed by the standard RNA deep sequencing (RNA-seq) as described in the material and methods. RNA-seq read density for TFAM transcripts was plotted with the relative RNA-seq read coverage (counts). Fragments per kilobase of exon per million fragments mapped (FPKM) were calculated to compare the expression level of TFAM mRNA variants in each sample.

TFAM / DAPI

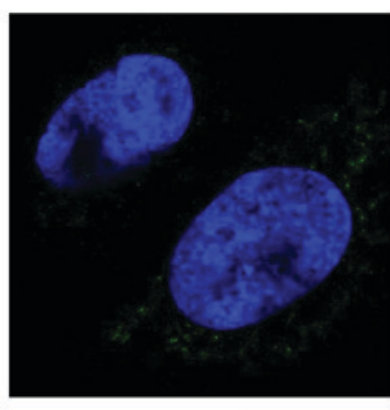

$10 \mu \mathrm{m}$
TFAM / DAPI

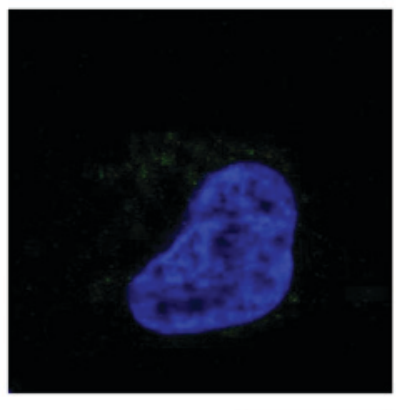

$10 \mu \mathrm{m}$

Figure 3. Subcellular localization of TFAM in U251-MG cell. U251-MG cells were grown on glass coverslips, fixed and permeabilized with $0.2 \%$ Triton X-100. After the immuno-staining of cells with anti-TFAM antibody, cover slips were mounted with Vectashield and visualized using a Zeiss confocal microscope. Scale bars, $10 \mu \mathrm{m}$.
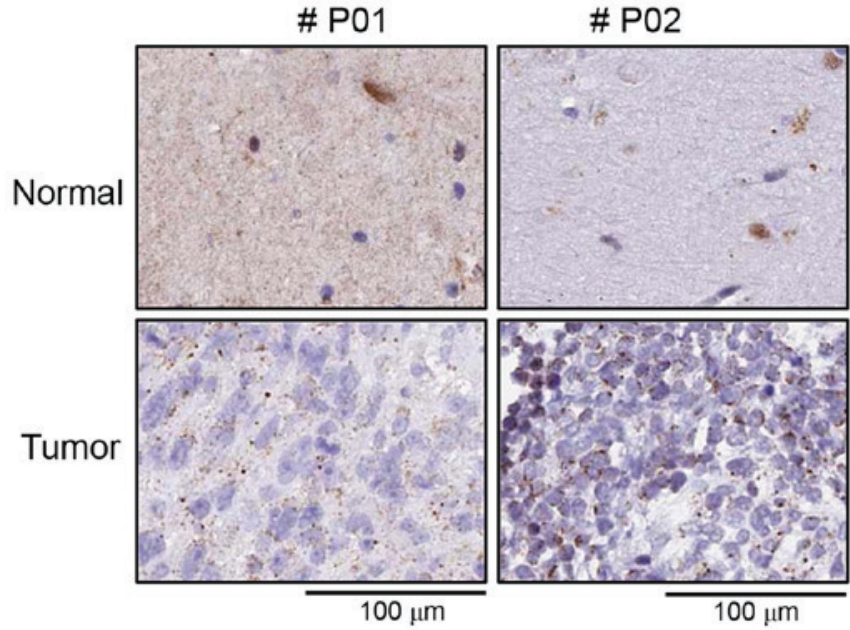

Figure 4. TFAM expression levels in human brain tumors. Human glioma tissue arrays were analyzed by immunohistochemistry (IHC) for TFAM staining Representative images from two patients were shown. Scale bars, $100 \mu \mathrm{m}$. 


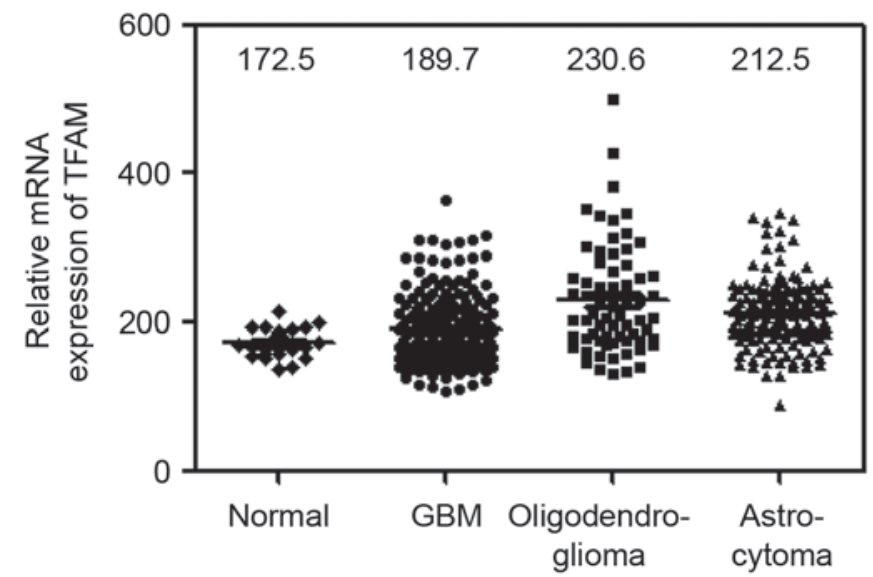

Figure 5. Differential expression of TFAM mRNA in glioma specimens of the REMBRANDT cohort. The expression levels of TFAM mRNA were detected in various glioma (normal, $\mathrm{n}=21$; $\mathrm{GBM}, \mathrm{n}=214$; oligodendroglioma $n=66$; astrocytomas, $n=145$ ). The median value of each group is indicated as a line.

Then mRNA were fragmented into small pieces, followed by the reverse-transcription of cleaved $\mathrm{mRNA}$ into the final cDNA library. Fragments per kilobase of exon per million fragments mapped (FPKM) were calculated to compare the expression level of TFAM mRNA variants in each sample. As shown in Fig. 2, FPKM values were markedly higher in U87-MG cells (24.9) and U251-MG cells (66.0) than its of cerebral cortex (8.3), indicating that TFAM is transcriptionally upregulated in glioblastoma cells.

Subcellular distribution of TFAM in U251-MG cells. In order to investigate the function of TFAM, the subcellular localization of TFAM were monitored in U251-MG cells by using immunocytochemistry. Interestingly TFAM is located to the dot-like structure close to nucleus, probably mitochondria (Fig. 3), suggesting that the TFAM is mainly located to mitochondria in glioblastoma cells.

Positive relationship between high TFAM expression and glioblastoma tissue specimens. In order to further elucidate the previous results (Figs. 1 and 2) in clinical setting, immunohistochemical analysis with human cancer tissue array were employed. As shown in Fig. 4, tumor tissue were well stained with anti-TFAM antibody compared to surrounding normal tissues, indicating that TFAM expression is upregulated in glioblastoma patients.

The differential mRNA expression and prognostic value of TFAM was further validated in the REMBRANDT cohort. To further evaluate the previous observation (Figs. 1-3), the differential expression and prognostic significance of TFAM in glioma of REMBRANDT cohort (http:// www.betastasis.com/glioma/rembrandt/) were analyzed. Consistent with above mentioned analysis including tissue microarray, western blotting and RNA-sequencing, TFAM gene expression was markedly increased in various glioma (GBM, $\mathrm{n}=214$; oligodendroglioma, $\mathrm{n}=66$; astrocytomas, $\mathrm{n}=145)$ than in normal controls $(\mathrm{n}=21$; one way ANOVA $\mathrm{P}<0.0001$; Fig. 5).

\section{Discussion}

Glioblastoma multiform (GBM) is the most aggressive and common brain tumor, accounts for about $30 \%$ of all brain and central nervous system tumors with poor prognosis. During the past 10 years, the 5-year survival rate of glioblastoma patients was only $2 \%$, and the average survival rate was 1 year (22). However, the diagnosis for glioblastoma has not developed for a certain period of time, due to the resistance against radiotherapy and chemotherapy (23). Therefore, the putative analytical biomarkers and potential molecular targets for gliomas are immediately required to deal with this disease.

In the present study, we have identified TFAM as a new prognostic marker for GBM, which was upregulated in GBM cell line and patients tissues. It has been well established that TFAM is required for mtDNA replication and transcription (14). TFAM has been reported to participate in the regulation of cell survival, proliferation and migration. Vascular smooth muscle cell proliferation is dependent on the upregulation of TFAM expression in damaged rat carotid artery (24). In addition, TFAM regulates p21 (WAF1/CIP1), a critical regulator of cell cycle progression, since TFAM depletion induced p21-dependent G1 cell cycle arrest (25). Moreover, TFAM is associated to the development and progression of malignant tumors. In this regard, TFAM mutation induced mtDNA depletion and apoptotic resistance in colorectal cancer (26). However, TFAM function in glioma is poorly understood. Therefore we have investigated if TFAM is potentially involved in brain tumor including GBM. Interestingly, TFAM was highly expressed in glioblastoma cell lines, including U343-MG cell and U373-MG cell (Fig. 1A). Analysis of qRT-PCR with TFAM-specific primer have indicated that U343-MG cell and U373-MG cells showed the higher expression of TFAM mRNA (Fig. 1B) compared to U87-MG cells. In addition, RNA deep-sequencing of U87-MG cell and U251-MG cells provide the evidence that FPKM of TFAM mRNA is higher in these cells compared to in normal brain (Fig. 2).

In order to further evaluate the possible function of TFAM in brain tumor, subcellular localization of TFAM were examined in U251-MG cells. As expected, TFAM is located to the dot-like structure close to nucleus, probably mitochondria (Fig. 3), suggesting that the mitochondria-located function of TFAM is essential for its role in glioblastoma cells. To further investigate the central role of TFAM in GBM development, tissue micro array were performed. As shown in Fig. 4, TFAM was upregulated in glioblastoma sample. By using clinical data from REMBRANDT cohort, TFAM is greatly expressed in all glioma including GBM (Fig. 5), clearly indicating that TFAM expression is somehow associated to the clinical prognosis of glioma. Taken together TFAM is appeared to be associated to glioma development in addition to be a new prognostic tool for glioblastoma.

\section{Acknowledgements}

This study was financially supported by research fund of Chungnam National University in 2014 (Jongsun Park) and by the National Research Foundation of Korea (NRF) grant funded by the Korea Government (MEST) (nos. NRF-2012 
M3A9B6055302, NRF-2014R1A1A3050752, NRF-2015 R1A2A2A01003597 and NRF-2015R1D1A3A01015694).

\section{References}

1. Friedmann-Morvinski D, Bushong EA, Ke E, Soda Y, Marumoto T, Singer O, Ellisman MH and Verma IM: Dedifferentiation of neurons and astrocytes by oncogenes can induce gliomas in mice. Science 338: 1080-1084, 2012.

2. Arif Arshad A, S.H: EGFR and PTEN gene mutation status in glioblastoma patients and their prognostic impact on patient's survival. J Carcinog Muta 6, 2015.

3. Virk SM, Gibson RM, Quinones-Mateu ME and BarnholtzSloan JS: Identification of variants in primary and recurrent glioblastoma using a cancer-specific gene panel and whole exome sequencing. PLoS One 10: e0124178, 2015.

4. Bergers $G$ and Benjamin LE: Tumorigenesis and the angiogenic switch. Nat Rev Cancer 3: 401-410, 2003.

5. Wesseling P, Ruiter DJ and Burger PC: Angiogenesis in brain tumors; pathobiological and clinical aspects. J Neurooncol 32: 253-265, 1997.

6. Jain RK, di Tomaso E, Duda DG, Loeffler JS, Sorensen AG and Batchelor TT: Angiogenesis in brain tumours. Nat Rev Neurosci 8: 610-622, 2007.

7. Das S and Marsden PA: Angiogenesis in glioblastoma. N Engl J Med 369: 1561-1563, 2013.

8. Carmeliet P and Jain RK: Molecular mechanisms and clinical applications of angiogenesis. Nature 473: 298-307, 2011.

9. Norden AD, Drappatz J and Wen PY: Novel anti-angiogenic therapies for malignant gliomas. Lancet Neurol 7: 1152-1160, 2008.

10. Chinot OL, Macdonald DR, Abrey LE, Zahlmann G, Kerloëguen Y and Cloughesy TF: Response assessment criteria for glioblastoma: Practical adaptation and implementation in clinical trials of antiangiogenic therapy. Curr Neurol Neurosci Rep 13: 347, 2013.

11. Chinot OL, Wick W, Mason W, Henriksson R, Saran F, Nishikawa R, Carpentier AF, Hoang-Xuan K, Kavan P, Cernea D, et al: Bevacizumab plus radiotherapy-temozolomide for newly diagnosed glioblastoma. N Engl J Med 370: 709-722, 2014.

12. Gilbert MR, Dignam JJ, Armstrong TS, Wefel JS, Blumenthal DT, Vogelbaum MA, Colman H, Chakravarti A, Pugh S, Won M, et al: A randomized trial of bevacizumab for newly diagnosed glioblastoma. N Engl J Med 370: 699-708, 2014.

13. Ekstrand MI, Falkenberg M, Rantanen A, Park CB, Gaspari M, Hultenby K, Rustin P, Gustafsson CM and Larsson NG: Mitochondrial transcription factor A regulates mtDNA copy number in mammals. Hum Mol Genet 13: 935-944, 2004.
14. Picca A and Lezza AM: Regulation of mitochondrial biogenesis through TFAM-mitochondrial DNA interactions: Useful insights from aging and calorie restriction studies. Mitochondrion 25: $67-75,2015$

15. Cheng $\mathrm{Z}$ and Ristow M: Mitochondria and metabolic homeostasis. Antioxidants \& redox signaling 19: 240-242, 2013.

16. Kim AY, Kwak JH, Je NK, Lee YH and Jung YS: Epithelial-mesenchymal transition is associated with acquired resistance to 5-fluorocuracil in HT-29 colon cancer cells. Toxicol Res 31: 151-156, 2015.

17. Li Y, Park J, Piao L, Kong G, Kim Y, Park KA, Zhang T, Hong J, Hur GM, Seok JH, et al: PKB-mediated PHF20 phosphorylation on Ser291 is required for p53 function in DNA damage. Cell Signal 25: 74-84, 2013.

18. Kim IS, Yang SY, Han JH, Jung SH, Park HS and Myung CS: Differential gene expression in GPR40-overexpressing pancreatic $\beta$-cells treated with linoleic acid. Korean J Physiol Pharmacol 19: 141-149, 2015.

19. Bioinformatics UG 2015: Human genome sequencing consortium. http://genome.ucsc.edu. Accessed April 17, 2016.

20. Kim D and Salzberg S 2015: TopHat 2.1.0 release 6/29/2015. https://ccb.jhu.edu/software/tophat/index.shtml.

21. Na CH, Hong JH, Kim WS, Shanta SR, Bang JY, Park D, Kim HK and Kim KP: Identification of protein markers specific for papillary renal cell carcinoma using imaging mass spectrometry. Mol Cells 38: 624-629, 2015.

22. Jiang J, Yang J, Wang Z, Wu G and Liu F: TFAM is directly regulated by miR-23b in glioma. Oncol Rep 30: 2105-2110, 2013.

23. Chargari C, Moncharmont C, Lévy A, Guy JB, Bertrand G, Guilbert M, Rousseau C, Védrine L, Alphonse G, Toillon RA, et al: Cancer stem cells, cornerstone of radioresistance and perspectives for radiosensitization: Glioblastoma as an example. Bull Cancer 99: 1153-1160, 2012 (In French).

24. Yoshida T, Azuma H, Aihara K, Fujimura M, Akaike M, Mitsui T and Matsumoto T: Vascular smooth muscle cell proliferation is dependent upon upregulation of mitochondrial transcription factor A (mtTFA) expression in injured rat carotid artery. Atherosclerosis 178: 39-47, 2005.

25. Kim AJ, Jee HJ, Song N, Kim M, Jeong SY and Yun J: p21 (WAF1/C1P1) deficiency induces mitochondrial dysfunction in HCT116 colon cancer cells. Biochem Biophys Res Commun 430: 653-658, 2013.

26. Guo J, Zheng L, Liu W, Wang X, Wang Z, Wang Z, French AJ, Kang D, Chen L, Thibodeau SN and Liu W: Frequent truncating mutation of TFAM induces mitochondrial DNA depletion and apoptotic resistance in microsatellite-unstable colorectal cancer. Cancer Res 71: 2978-2987, 2011. 